IOSR Journal of Computer Engineering (IOSR-JCE)

e-ISSN: 2278-0661, p-ISSN: 2278-8727Volume 16, Issue 2, Ver. XII (Mar-Apr. 2014), PP 54-66

www.iosrjournals.org

\title{
A Systematic Combined Approach to Prolong the Life Time of Heterogeneous WSN without Compromise the Performance
}

\author{
Aminur Rahman ${ }^{1}$, Rajbhupinder Kaur ${ }^{2}$ Sanjib Das ${ }^{3}$ \\ ${ }^{1}$ Research Scholar, ${ }^{2}$ Faculty of Engineering, ${ }^{3}$ Technology Analyst \\ ${ }^{1,2}$ Department of Computer Engineering, Yadvindra College of Engineering, Punjabi University Guru kashi \\ Campus, Talwandi Sabo, Punjab, INDIA \\ ${ }^{3}$ Infosys Technology, Pune, Maharashtra, INDIA
}

\begin{abstract}
Having seen the robust advantages of Wireless Sensor Networks' applications, many research works has been done to prolong the life time of the network. The objective of our research work is to find out a novel approach for synergetic improvement into the life time of WSN. Most of the approach mainly used only single technique to increase the life. In our research subject we have searched that "Is the systematic combination of more than one technique can provide more improvement?" In this paper we have proposed "A Systematic Combined Approach" which combines multiple static and dynamic techniques. Increasing the life time of the network is based on lower energy consumption and higher degree of balanced consumption of energy. Lower energy consumption can be achieved by reducing the volume of data and the distance to be travel by data. This approach uses some powerful data processing node in the dense area of sensor nodes to reduce the volume of data. Optimal location of the base station, typel relay nodes and type 2 relay nodes are used to reduce the Euclidean distance. Finally, we have applied a newly designed protocol "Average Energy Dynamic Clustered Protocol (AEDCP) as a dynamic technique for balanced consumption of energy. We have implemented this approach on a network simulator NS2 and compare the results with similar BEEGP (Balanced Energy Efficient Grouping Protocol), and we have found that the proposed approach is more energy efficient without compromising the performance.
\end{abstract}

Keywords: Systematic Combined Approach, Data Processing Nod, Heterogeneous Wireless Sensor Network, Relay Node, Network Life Time, Type 1 Relay Node, Type 2 Relay Node.

\section{Introduction}

Wireless Sensor Networks are composed of a lot of small, low cost sensor nodes that work together to measure various parameters of the environment and send the data to a unique or several sinks where they will be processed. WSNs have a wide range of uses in military, medical, metropolitan and industrial venues [7]. The majority of these applications may be split into two classifications: data collection and event detection.

Although reducing the size of sensors mote could make them cheaper, this also requires that all hardware equipment, especially batteries, to be very small. So, each node has very limited resources in terms of processing speed, storage capacity and bandwidth. In addition, their lifetime is determined by their ability to conserve power. So, these limitations are a significant factor and must be addressed when designing and implementing a wireless sensor network for a specific application. Since the sensor motes should be functional for a long period of time and battery replacement in harsh environments like battlefields is usually difficult, nodes may lose their energy very fast, thus becoming non-functional in a short duration. This situation can affect the network connectivity, tolerance and lifetime.

When a wireless sensor network is need to be employed for event detection, such as detecting the ignition of a fire, it would be anticipated that the sensor nodes must remain awake thus consuming their precious limited power [7]. Therefore, optimization for energy consumption is an important issue, especially to increase lifetime in WSN.

To address this problem, a variety of approaches are implemented in the area of network layer, physical and also in combined layer. Neighbourhood energy depletion problem is one major problem can which create the network partitioning.To solve this problem many technique like use multiple sinks, use of another solution is mobility (sink mobility and neighbour mobility) has been done in the previous work .Besides of this problem another problem that using a single optimal path for every communication may gradually drain the energy of nodes which are located on the route. This cause arises some problems such as node and link failure due to unbalanced depletion of nodes' batteries across the network. Applying multi-path routing in WSNs could result in traffic and energy load balancing over the network. Further, it is not necessary to update the route information time to time, which wastes a high amount of the nodes' power. If, the sensor nodes forward the data and control packets to the next hop at a maximum power level, which results in fast energy exhaustion. In this situation, by 
employing a power control scheme in routing protocols in which the nodes are able to adjust the transmission power level based on the distance from the next hop, the relay nodes can conserve much energy. Biologically inspired algorithms can optimize the route construction phase for optimum distance.

Our aim in this paper is to help readers better understand the various problems; those have been standing as hinders in the prolonging of life time of WSN. Many previous research works has focused only one problem or used only one technique. In this paper, we disclose the potential of Systematic Combination of more than one technique for improving network lifetime. We have also listed many approaches on the basis of some issues; those have great contribution reduce the energy consumption. This includes the reducing Euclidean distance, reducing volume of data, Power control and increasing the degree of balance energy consumption, computational complexity, and constrained about parameter. To, the best of our knowledge, our work is the first effort to listed lifetime improvement strategies applied in energy consumption and balanced consumption of energy for wireless sensor network.

The rest of this paper is organized as follows: We present the literature Review in section 2. Section 3 presents Problem Statement, Objectives, Parameters, Problem Formulation and also assumption. Section 4 presents about proposed approach and methodologies. A brief description of implementation platform and results of proposed approach has presented in Section 5. Section 6 present the discussion about the various result.And finally in the Section 7 we conclude that our approach is more energy efficient and there are many future scope of this work.

\section{Literature Review}

Lifetime improvement mechanisms in routing protocols for WSNs are basically divided into two main categories: simultaneous schemes and cross-layer schemes. Simultaneous schemes usually refer to the mechanisms which could be combined with routing algorithms in order to achieve a specific goal like energy efficiency. In WSNs, these techniques are classified based on the protocol operation. However, cross-layer schemes investigate different layers simultaneously to make the protocol more energy-efficient.

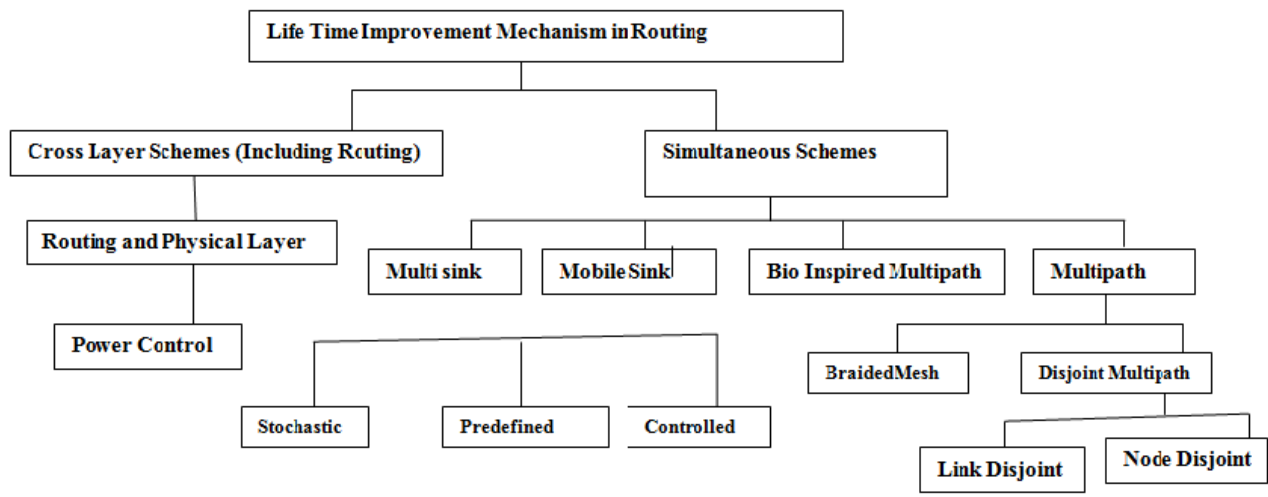

Fig 1.a. Classification of lifetime improvement mechanisms [7]

Many energy-efficient mechanisms categories such as multi-sink, mobile sink, multi-path, bio-inspired and power control (as a cross-layer approach) technique [7] [8]. One thing has been observed that many of the algorithms only focuses on only life time improvement mechanism for prolonging the life time but not about the constrained parameters.

\subsection{General classification routing protocols}

On the basis network structure, routing protocols of Wireless Sensor Network can be divided into three categories. Data Centric, Hierarchical and Geographic [7]

i. Data-Centric Protocols: Multi-hop data-centric routing protocols are basically the first class to be introduced in WSN. Considering a large number of nodes in sensor networks, flat algorithms employed query based technique, in which the sink node only requests the desired data in order to prevent continuous data transmissions and thus save power. In this, Sensor Protocols for Information via Negotiation (SPIN) [8], Directed Diffusion [8], EnergyAware Routing (EAR) [17], Rumours Routing [17] and Minimum Cost Forwarding Algorithm (MCFA) [7] are some of the most famous flat algorithm paradigms.

ii.Hierarchical Protocols: Hierarchical protocols utilize a clustering scheme; nodes are assigned different roles. Energy conservation can be achieved in these protocols by some aggregation and reduction of data in so-called cluster heads (CHs). In this class, Two Tier Data Dissemination (8), Low-Energy Adaptive Clustering Hierarchy (LEACH) [7][8], Threshold-Sensitive Energy-Efficient Sensor Network Protocol (TEEN) [7], Adaptive 
Periodic Threshold-Sensitive Energy-Efficient Sensor Network Protocol (APTEEN) [7] and Power-Efficient

Gathering in Sensor Information Systems (PEGASIS) [7] are some inspiring protocols.

iii Location Based Protocols: The possibility to apply position information in routing schemes will be used in location-based algorithms to route data towards the desired regions in the sensor field. It can save energy by limiting the flooding through the network. GPSR, GAF and GEAR fall in this class [7].

\subsection{Classifications of Protocols based on reducing distance, volume of data, and degree of balance energy consumption and constrained parameter.}

\begin{tabular}{|l|l|l|l|l|l|l|l|}
\hline $\begin{array}{l}\text { SL. } \\
\text { No. }\end{array}$ & $\begin{array}{l}\text { Name of Techniques } \\
\text { / Reference }\end{array}$ & $\begin{array}{l}\text { Optimal } \\
\text { distance }\end{array}$ & $\begin{array}{l}\text { Volume } \\
\text { of Data }\end{array}$ & $\begin{array}{l}\text { Power } \\
\text { control }\end{array}$ & $\begin{array}{l}\text { Balanced } \\
\text { Consumption }\end{array}$ & $\begin{array}{l}\text { Computational } \\
\text { Complexity }\end{array}$ & $\begin{array}{l}\text { Constrained } \\
\text { parameters }\end{array}$ \\
\hline 1 & Ref[1] & Yes & No & No & Yes & Moderate & No \\
\hline 2 & Ref [2] & No & Yes & No & No & low & No \\
\hline 3 & Ref [3]-IEEABR & No & No & No & Yes & High & Yes \\
\hline 4 & Ref [4]-BEEG & No & No & No & Yes & Moderate & No \\
\hline 5 & Ref[5] -Relay & Yes & No & No & No & Moderate & No \\
\hline 6 & Ref [6] -Heterogeneity & No & No & No & Yes & Moderate & Yes-( High) \\
\hline 7 & Ref [8]-Tiny Raged & Yes & No & No & Yes & High & No \\
\hline 8 & Ref [8]-TSEP & Yes & No & No & Yes & Moderate & No \\
\hline 9 & Ref [8]-GAAC & Yes & No & No & Yes & High & No \\
\hline 10 & Ref [10]-FCA & Yes & No & No & Yes & Moderate & No \\
\hline 11 & Ref[11]ACO-MSS & No & No & No & Yes & Moderate & No \\
\hline 12 & Ref[13]-CPSP & Yes & No & Yes & No & low & No \\
\hline 13 & Ref[14] -Optimal & Yes & No & No & No & High & No \\
\hline 14 & Ref[15] & Yes & No & No & No & low & No \\
\hline 15 & Ref[18]-EECAM & Yes & No & No & Yes & Moderate & No \\
\hline 16 & Ref [20]-Multilink & Yes & No & No & Yes & High & No \\
\hline 17 & Ref [21]Compression & & Yes & No & & Moderate & Yes \\
\hline 18 & Ref [25] MOM, BASE & Yes & No & No & No & High & Yes(High) \\
\hline
\end{tabular}

Table 2.a. Classifications of Protocols based reducing distance, volume of data, and degree of balance energy

\subsection{Protocols using multiple mechanism}

The table listed some protocols and their metrics, those proposed and simulated by many scholar showed that, it can give more prominent result than using single technique.

\begin{tabular}{|c|c|c|c|c|c|c|c|c|}
\hline \multirow{2}{*}{$\begin{array}{l}\text { Protocol } \\
\text { /Reference }\end{array}$} & \multirow[t]{2}{*}{ Mechanism } & \multicolumn{7}{|c|}{ Metrics of Different Protocols } \\
\hline & & $\begin{array}{l}\text { Sink } \\
\text { movement }\end{array}$ & $\begin{array}{l}\text { Sink Speed/ } \\
\text { Mobility }\end{array}$ & $\begin{array}{l}\text { Location } \\
\text { Awareness }\end{array}$ & $\begin{array}{l}\text { Number } \\
\text { of Sink }\end{array}$ & $\begin{array}{l}\text { Network } \\
\text { Structure }\end{array}$ & $\begin{array}{l}\text { Aggre- } \\
\text { gation }\end{array}$ & $\begin{array}{l}\text { Applicatio } \\
\text { n Type }\end{array}$ \\
\hline MSDD [32] & $\begin{array}{l}\text { Multi sink, } \\
\text { Multi path }\end{array}$ & N/A & N/A & No & $\mathrm{K}(\mathrm{K} \geq 2)$ & Flat & Yes & $\begin{array}{l}\text { Query } \\
\text { Driven }\end{array}$ \\
\hline $\begin{array}{l}\text { MSLBR } \\
{[16]}\end{array}$ & $\begin{array}{l}\text { Multi sink, } \\
\text { Multi path }\end{array}$ & N/A & N/A & No & $\mathrm{K}(\mathrm{K} \geq 2)$ & Flat & No & $\begin{array}{l}\text { Time } \\
\text { Driven }\end{array}$ \\
\hline SMPD[17] & $\begin{array}{l}\text { Multi Sink } \\
\text { \& Path }\end{array}$ & Random & Constant & $\begin{array}{l}\text { Yes, } \\
\text { Only Sink }\end{array}$ & $\mathrm{K}(\geq 1)$ & Flat & No & $\begin{array}{l}\text { Time } \\
\text { Driven }\end{array}$ \\
\hline [19] & $\begin{array}{l}\text { Multi \& } \\
\text { Mobile Sink }\end{array}$ & Fixed, & mobile & Yes, & $\mathrm{K}(\geq 1)$ & Flat & No & $\begin{array}{l}\text { Time } \\
\text { Driven }\end{array}$ \\
\hline [25] & $\begin{array}{l}\text { Multi sink, } \\
\text { Mobile Sink }\end{array}$ & $\begin{array}{l}\text { Controlled } \\
\text {,Random }\end{array}$ & & $\begin{array}{l}\text { Yes, } \\
\text { Only Sink }\end{array}$ & $\mathrm{K}(\geq 1)$ & Flat & No & $\begin{array}{l}\text { Time } \\
\text { Driven }\end{array}$ \\
\hline MSRP [28] & $\begin{array}{l}\text { Mobile \& } \\
\text { Power } \\
\text { control }\end{array}$ & Controlled & Adaptive & $\begin{array}{l}\text { Yes, } \\
\text { Only Sink }\end{array}$ & 1 & $\begin{array}{l}\text { Hierarchic } \\
\text { al }\end{array}$ & Yes & $\begin{array}{l}\text { Not } \\
\text { Specified }\end{array}$ \\
\hline
\end{tabular}

Tale 2.b. Showing the protocol combining much mechanism and their metrices [7] 


\subsection{Systematic explanation of problems and positive scope of existing techniques}

i. Limited Transmission Range: Sensor nodes are placed in some particular location of the area of attention. As sensor nodes have limited transmission range, only placed senor nodes are may not be sufficient to construct network to send their data to Base Stations.

ii. Density of node distribution: Many research articles consider that sensor nodes are equally distributed for the convenient of simulation [1]. But practically we will have hardly equally distributed sensor network. So, it will be very better to consider the unequal distribution of sensor nodes.

iii. Location of the Base Station: As the energy consumption is linear function of distance travel by any data packet. So, definitely the location of the base station has a great impact on the energy consumption. [14].So, here the problem is to find out the best suitable location of the Base Station. It is a NP problem. So it is a little bit difficult to find out best single appropriate solution.

iv. Homogeneous and Heterogeneous Sensor Network: Many of previous algorithms considered the WSN as homogeneous [30]. But, in practical scenario WSN consist of many heterogeneous nodes. So all the algorithm made for homogenous may not work efficiently in all practical scenario so, here is a need of some robust algorithm, which can work more energy efficiently without compromising the performance. Many algorithms those are developed for homogenous WSN and also some, those developed for heterogeneous WSN does not take in to accounts the different bandwidth of Link between different nodes. If we consider it, then it will be more practical [6].

v. Techniques for Reduce the distance and volume of data: To reduce the volume of data and distance many algorithm has been developed. Among those, some algorithm consider clustered network. The entire node connecting to the clustered head will send their data to the clustered head to reduce their travelling distance and to reduce their volume of data. Very famous algorithm known as LEACH also comes under this category. In this algorithm acting as role of cluster head have need more energy consumption. To reduce this problem although LEACH algorithm changes the role of Cluster head within the cluster among the node after some round based on residual energy. Although clustered network architecture have more advantages as compared to flat network architecture in distance parameter, even none exiting single algorithm can reduce the distance and volume of data simultaneously and these require more computational process and reducing of volume of is not satisfactory.

vi. Hotspot problem: To tackle out the hotspot problem, many algorithms have been developed, but none single algorithm showed satisfactory result. One of algorithm comes which considered the use of mobile sink [11]. According to this algorithm the sink will fly from one location to another predefined or random location and during fly and it will not communicate with any node .But, the idea of flying is not practical, because in some critical application area, flying of Sink above the forbidden region may be visible to the enemies. As it will not communicate during the flying, it can't give response to any event occurs immediately. So for real time system this idea will not practical. Another algorithm Fuzzy Clustering Algorithm (FCA) is approach to overcome the hotspot problem occurred in LEACH protocol [10]. FCA adjusts the cluster-head radius considering the residual energy and the distance to the base station parameters of the sensor nodes. This help to decrease the intra cluster work of the sensor nodes which are closer to the base station or have lower battery level. To solve this problem we can also use multiple sink. But again the problem is to determine the suitable location of the respective location of the sink. Although it has provides a little improvement in energy efficiency, but it is again a costly mechanism

vii. One hope vs. multi hop communication within a Cluster: In all the clustering algorithm one very important issues is to taken whether Single Hop Communication and Multi hope communication. The LEACH protocol and many of the variants LEACH Protocol considered on hope communication by the data of node to reach the clustered head. In "Homogeneous vs. Heterogeneous Clustered Sensor Network: A Comparative Study" [30], have explored that multi-hope communication is more energy efficient and also in the term of hardware cost. In [30-] the author at first compared the single hope homogeneous LEACH with the variant M-LEACH (Multi hop), where he found that M- LEACH is better than original LEACH. Finally M-LEACH (homogeneous) is also compared with the heterogeneous M-LEACH. Here the author found that in maximum case heterogeneous M-LEACH outperforms than original LEACH and also homogeneous M-LEACH. So in our proposed approach, we can take into account this feature to add outperformance.

viii.Biologically inspired algorithm: Many biologically inspired algorithms have developed to solve various real life problems. Similarly ANT Algorithm, Ant Optimization Algorithm and Improved Algorithm, Jumping Ant Algorithm have been proposed for the WSN to find out best shortest path, to balance the traffic overhead of the shortest path providing less traffic alternative path and to easily tackle out the failure of nodes automatically, and to capture the dynamic changing topology.[3]Although these algorithms have many advantages, but one disadvantage is that a large amount time is consumed to find out the shortest path, during that many packet will travel at random via many long rout, as a result the energy will be high. So we have a 
new idea to solve this problem. To tackle out the failure of node and to reduce the problem of heavy traffic in shortest path, one idea is to keep option of different address of different adjacency nodes those within the range of sensor nodes in the routing table of each node.

ix. Optimum number and location of Relay node: Since, energy consumption $\mathrm{Ec} \propto \mathrm{E}_{\mathrm{d}}$ (Euclidean distance), So many algorithm have designed to reduce distance to be travel by data with different approach approaches. One algorithm come optimal placement of relay node [29] to reduce distance travel by the data. Another algorithm argues that adding more than an optimum numbers relay has no effect on energy saving [15]. So it is very important to consider the number and the location of relay node to construct an energy efficient sensor network Structure.

\section{Problem Statement, Objectives, Parameters and Problem Formulation}

\subsection{Statement of the problem}

There are enormous problem associated with Wireless Sensor Network due its diverse application areas. Many different problems arise in different application areas of wireless sensor network. But, common problem and big issues of WSN in all application areas is short life time of wireless sensor node and issue is how can increase the life time of network by increasing the life time of node. Here we will state only those problem of WSN and drawbacks of exiting techniques which hinders in prolonging of network life time.

Transmitting of redundant raw data, Isolation of node, Short Route/ Not consideration of optimal location of Base Station, Hot spot problem, Network partitioning problems, Imbalance Energy consumption, Parameter to measure the degree of balance energy consumption, Not consideration of Important Junction Node, Total life time of the network is measured in the terms round number where all nodes dies and Not consideration of Sleep and Active mode is application specific.

\subsection{Objectives}

Main objective our research work is to prolong the life time of the network. To achieve this objective, we have considered the following other important objectives.

i. To reduce rate of total energy consumption after each round.

ii.To increase the stability of the network ( Time up to first node failure)

iii. To increase the degree of balance energy consumption.

iv. To decrease the number of Residual alive nodes

v.To increase throughput

\subsection{Parameters}

We have taken five objectives parameter to measure the performance of our approach and these parameters are subjected to the constrained parameter, which are also given bellow. For example, we can't reduce the average energy consumption to a low values where packet drop fraction is unacceptable.

\subsubsection{Objective parameters}

i. Average Energy Consumption with respect to each round

ii. Average Residual Energy with respect to each round

iii. Number of live node with respect to each round

iv. Round number at which First Node Die

v.Degree of Balanced Energy consumption

\subsubsection{Constrained parameter}

\section{i. Average end to end delays ii.Throughput iii.Packet Drop Fraction(pdf)}

\subsection{Assumptions}

Different types of node has different energy level, different computation and processing power and also different range.

i. The band width of link between two tiers is different.

ii. Network architecture is hierarchical instead of flat.

iii. Transmission range is different of different types of nodes.

iv. All the sensor nodes are uniform but unequally distributed through the network.

v. Impact of taking the optimum transmission range has not taken into consideration.

vi. Data processing operation can be Aggregation, Sensory Fusion, Encryption, and Compression etc.

vii. We have consider heterogeneous network as heterogeneity is more energy efficient than homogeneous [30] viii.Active and Sleeping modes of various nodes is application specific to provide energy efficiency [13] 


\subsection{Problem formulation for objective function}

Energy Consumption by a node $\mathrm{E}_{\mathrm{c}}$ is the function of, Volume of data to be processed $\left(\mathrm{V}_{\text {data }}\right)$, Numbers of Operation to be performed before send data to the others node $\left(\mathrm{N}_{\mathrm{op}}\right)$, Euclidean distance between the two nodes $\left(E_{d}\right)$ and Energy loss due to the thermal agitation of electrons ( $\left.E_{l o s s}\right)$.

If $\uparrow$ denote the desirable value is high and $\downarrow$ denotes the desirable value is low, Energy Consumption Function, $\mathbf{E}_{\mathbf{c}} \downarrow=\mathbf{E}_{\mathrm{d}} \downarrow$ and $\mathbf{V}_{\text {data }} \downarrow$, and $\mathbf{N}_{\mathrm{op}} \downarrow$, and $\mathbf{E}_{\text {loss }} \downarrow$ (Eq.1) So, increasing of the life time of network is based on mainly two things a). The Energy Consumption Function Ec should as low as possible and b). Should has a high degree of Balance Energy Consumption [(d (BEC))].

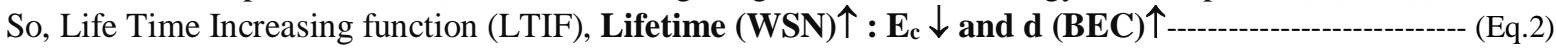
So, the objective function for increasing the life time of the network is to have lower value of Ec and high value of d (BEC). The LTIF also can be called as objective function. So, from the above Energy Consumption function and objective function we will have to understand that, any approaches we apply for the increasing of the life time of network should have capability to ,Decreases the Euclidean distance between two nodes ( $\left.E_{d} \downarrow\right)$, Reduce the volume of data to transmit from source to base station $\left(\mathrm{V}_{\text {data }} \downarrow\right)$, Reduce the number of operation to be perform for aggregation, conversion, and compressing $\left(\mathrm{N}_{\mathrm{op}} \downarrow\right)$, Provides high degree of Balance Energy Consumption .Energy loss is depending on quality of used radio equipment, so it is underlying area of Physical Layer. To reduce the Energy loss we will have use technically sound radio equipment. Now, degree of balance energy Consumption, $\boldsymbol{d}(\boldsymbol{B E C})=1 /$ Average of Average Deviation of Energy Consumption----------- (Eq.3)

\section{Methodologies of Proposed Approach}

The methodology of our proposed approach take advantage of some existing work. Our proposed approach is "Systematic Combined Approach (SCA)" which combines many existing idea with our own idea. This Combined Approaches means "Use of Powerful Data Processing Node in highly dense area of Sensor Nodes applying "Scanning of Square Partion Algorithm (SSPA)", and taken into account the optimal location of the Base Station applying the algorithm Minimum Average Distance of Base Station (MADBS) from all pdp nodes, and use of Type 1 Relay node applying SMTAR1( Steiner Minimum Tree Algorithm for Sensor Nodes) algorithm to construct Steiner Minimum Tree to ensure no node is in isolation and every senor node will transmit data to adjacent node through shortest distance, Type 2 Relay nodes are used to ensure that any remote pdp node will transmit data to base station only via pdp node or type2 relay node, and use of Average Energy Dynamic Cluster Protocol $(A E D C P)$ for balanced consumption of energy. This protocol constructs a hierarchical clustered network based on the average of initial energy with respect to each cluster and later when any cluster head changed, it goes to set up the new cluster on the basis of average residual energy. In this approach we proposed a new idea include a special type of node in the cluster for many data processing tasks to reduce the volume of data, to reduce the frequency of changing cluster head. We will compare this approach with existing protocol BEEGP (Balanced Energy Efficient Grouping Protocol) [4] to realize the energy efficiency.

\subsection{Steps of Systematic Combined Approach}

Step 1: Consider network area, number of nodes and their co-ordinates as unequally distributed

Step 2: Find out the required number and location of Powerful Data Processing Node

Step 3: Find out the optimal location of Base Station.

Step 4: Find out Required Number and location of Relay Node Type 2.

Step 5: Find out Required Number of Relay Node Type 1 to ensuring no node is in isolation.

Step 6:AEDC protocol to start data transmission and changing the cluster and cluster head

In the Step1 of the complete approach, initially we consider the Area of sensor network, Number of nodes and Co-ordinates Sensors nodes. Sensor nodes are unequally distributed in the area of attention. Some sensor nodes are may be in isolation. Type 1 relay nodes are use to solve this problem in step 5 using SMTR1 Algorithm.

4.1.1. Powerful Data Processing Nodes (step 2): The pdp nodes in some highly dense sensor area to reduce processing task of each sensor node before forward to the Base Station via Multi hop network. The number of required PDP node and about their co- ordinates should be taken as before the deployment of the network. Now the problem is that how can we can know where we Will have to Place the PDP node, what is the condition? We have proposed one idea that the whole area is to divide into equal square partion and find out the total number of nodes in that square partition. If total number of node is more than or equal to fixed (pre decided number) than place a PDP node in the center of that square partion or in a best location within that partion. We named this algorithm as "Scanning of Square Partition Algorithm" (SSP Algorithm). 
4.12. Optimal Location of Base Station (step 3): To find out a suitable location of base station we proposed an algorithm as "Minimum Average Distance of Base Station" (MADBS) from all pdp nodes. This algorithm will calculate average of total Euclidean distance for many alternatives location of Base Station. And finally decide that location, for which location algorithm return the minimum average distance of base station from all PDP nodes.

4.1.3. Types 2 Relay nodes (step 3): Type 2 Relay nodes are used to have optimal shortest path from all pdp nodes to base station and to ensure that any remote pdp node transmit data only via other pdp node or type 2 relay node. We do it by applying with the Steiner Minimum Tree Algorithm on pdp nodes. This idea of SMT was first proposed by Jakob Steiner for Combinatorial optimization Problems [33]. Ying Zhu and Qi Han also mention about construction of Steiner Minimum Tree to have optimum number of relay [15] node. The transmission range of Type relay nodes is equal to the transmission range of pdp nodes. We named this algorithm as SMTR2 Algorithm.

4.1.4. Relay Nodes (step 4): These are used to construct a transmission structure applying Steiner Minimum Tree Algorithm which will ensure the require minimum number of type 1 relay node to ensure no node is in isolation and every sensor node transmit data to short distance adjacent node. We named this algorithm as SMTR1 Algorithm.

4.1.5. Application of Average Energy Dynamic Clustered Protocol (step 5): This algorithm consists of Clustering Phase and Data Transmission Phase. It will dynamically construct a full-fledged Network by finding a Confirmed and Complete adjacency list each node in whole network. During the clustering Phase of this protocol routing table will be constructed for Forward and backward communication and it will be stored in each node. It is automatically done by the joint contribution of CMR message (Cluster Member Request Message) and JR (joint Request Message). The actual data transmission session start here in round wise, after every round, base station update the residual energy value of all nodes and Cluster Header will update the Residual energy of Cluster mebers, such that decision for the change of cluster head can be taken locally and decision for goes to next cluster setup phase monitored by the base station. Advantage of AEDCP: This provides the shortest path to it group member to transmit data to it, because group head accept the request those node whose acknowledgement get first. Since distance $\propto$ time, with constant speed, so Cluster Header will consider those nodes as member, those very nearby of the Cluster Header.

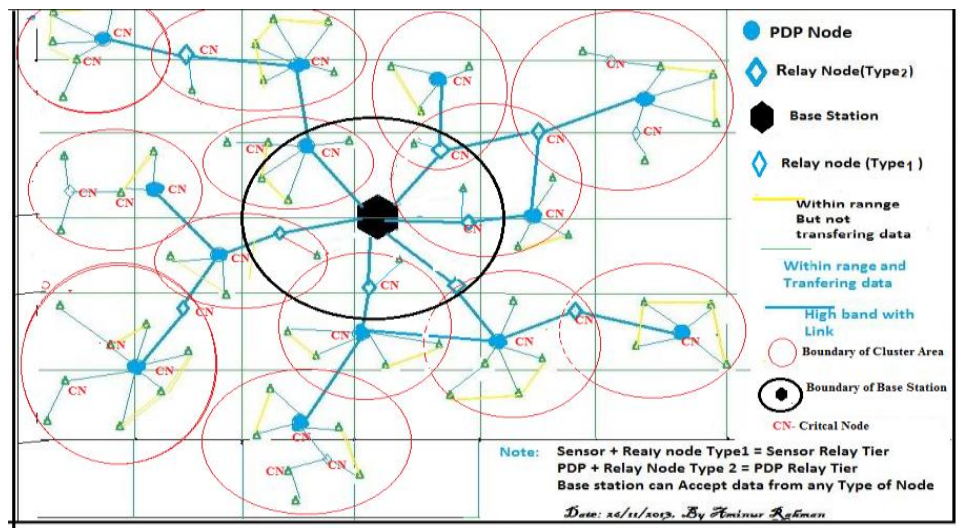

Fig 4.a. Showing the expected architecture of the network during the clustering and data transmission period

\section{2. Algorithm of AEDC Protocol: Step 5 of proposed approach in details}

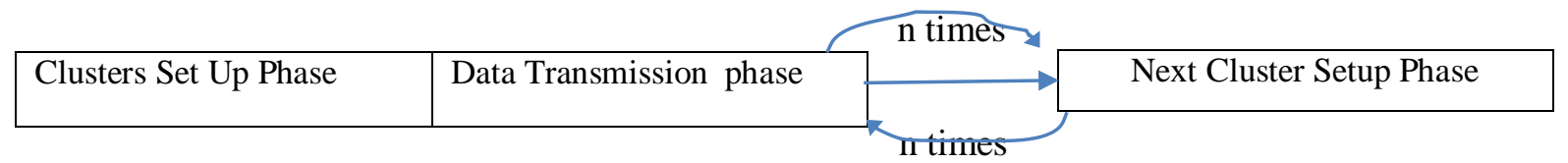

Step 1: The Base station will initially calculate Total Energy (TIE) using following formula. And it will also calculate the AIE (Average initial Energy) of each cluster

Step 2: Cluster Head Selection: Base Station will multicast a $\mathrm{CH}$ (Clustered Head) message to the entire PDP node to confirmed pdp node as cluster head.

Step 3: Set up Clusters by collecting cluster member based on Average Initial energy

3.1: Clustered Head will broad Cast CMR message to all the nodes for collecting Cluster member.

3.2: Routing table Constructed or change by CMR message and Fixed by JR message. 
3.3: Keep facility of alternative path for conditional alternative communication (CAPC) in during step 4 to avoid network partitioning

Step 4: Data Transmission Phase for transmit data in round wise

4.1: Calculate the residual Energy of every node after each round.

4.2: Compare Residual energy of cluster member with the residual energy of respective clustered head.

Step 5: If the residual energy of any cluster member is equal to or less than the energy of any other member,

5.1: Then the current clustered head will be ready to release TTBCH (Token to Become Clustered Head) token to transfer the role of cluster head to other member. Current Clustered Head will compare the residual energy of each member one by one, cluster head will release TTBCH token to that member which have highest residual energy

5.2: When any cluster head has been changed respective Cluster Header send TTNCS (time to next cluster set up) message to the Base Station.

Step 6: When TTNCS message has received by Base Station, It will call the Cluster Setup to set new clusters for all cluster head based on the Average Residual Energy.

6.1: Base station message SNC (Setup New Cluster) message

6.2: Base Station will calculate the total residual Energy (TRE) and calculate the Average residual Energy.

6.3: Go to step 3 and follow up to Step 6.

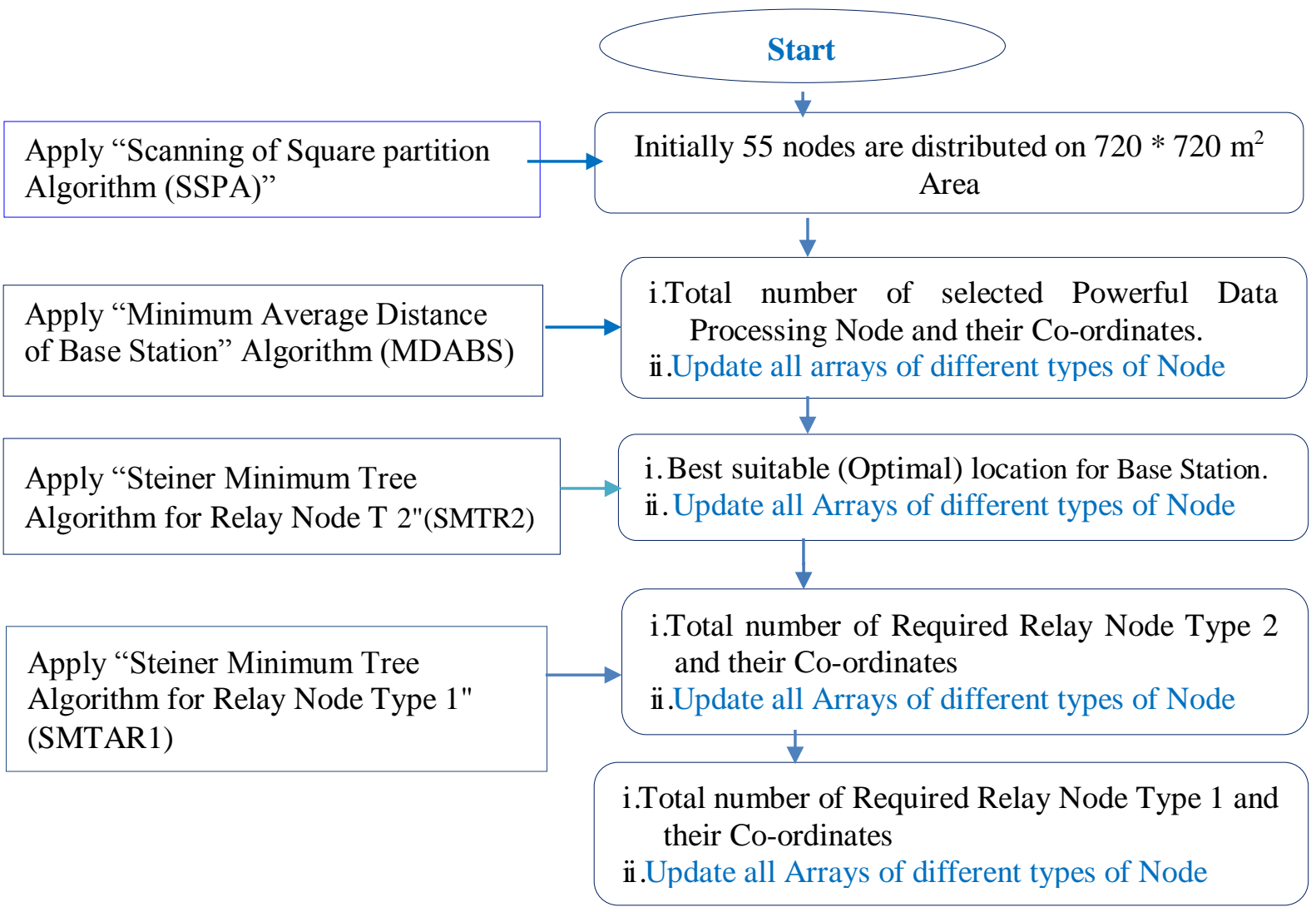

Apply “Average Energy Dynamic clustered Protocol” (AEDCP)
Complete Static Structure of the Network containing Sensor nodes, PDP nodes, Relay Node T1, T 2 and Base Station location

Starting of Clustering Phase, Data Transmission and Next cluster Set up Phase up to end of the Network life Time

\section{End}

Fig 4.b. Showing the flow of various steps of Systematic Combined Approach 


\subsection{Network Model and Radio Parameters}

\section{Implementation and Result}

In our approach we have taken $720 * 720$ square meter area. Initially, we have taken sensor node 5 sensor node random and uniformly distributed through the Network. Initially Location of the Base Station is not determined, we find out the best optimal location using MADBS algorithm. The node sense data at a fixed rate Transmit data to the Base Station, either directly to the Base Station, or via PDP node ; that using multi hop communication. The number of Cluster or Cluster Head is equal to the number of PDP node and this will have to be determined applying Scanning of Square Partion Algorithm. Similarly the number and Coordinates of Type 1 and Type 2 Relay node will be determined by applying SMTR1 and SMTR2 Algorithm.

We assume, the transmitter consume energy to run the radio electronics and Power amplifier. The receiver also consumes energy to run radio electronics. When the node transmit a message of $l$ bits through distance $d$ the node expends Energy $E_{T x}(l, d)$ energy and

$$
\begin{gathered}
E(l, d)=l^{*}{\underset{O r}{\text { lect }+}}_{\boldsymbol{O r}} l \varepsilon_{\mathrm{s}} d^{2} \text { for } \mathrm{d}<\mathrm{d}_{0} \\
E(l, d)=l^{*} E_{\text {lect }+} l \varepsilon_{m p} d^{4} \text { for } d>d_{0}
\end{gathered}
$$

\begin{tabular}{|c|c|c|c|c|c|}
\hline $\begin{array}{l}\text { SL. } \\
\text { No }\end{array}$ & Symbol & Parameters Name & $\begin{array}{l}\text { SL. } \\
\text { No }\end{array}$ & Symbol & Parameters Name/value \\
\hline 1 & Area $=700 * 700$ & Length $*$ Breadth $=$ Area & 10 & $\mathrm{IE}_{\operatorname{trn} 2=5}$ joule & Initials Energy Relay T2 \\
\hline 2 & $\mathrm{r}=20$ meter & Range of Senor Nodes & 11 & Base Station Energy & Sufficient Energy (Assume) \\
\hline 3 & r1=20 meter; & Range of relay node Type 1 & 12. & Data Size & 4000 bits \\
\hline 4 & $\mathrm{r} 2=30$ meter & Range of relay node Type 2 & 13 & Message Size & 100 bits \\
\hline 5 & $\mathrm{R}=30$ meter & Range of PDP node & 14 & Eelect & $50 \mathrm{~nJ} / \mathrm{bit}$ \\
\hline 6 & $\mathrm{BR}=30$ meter & Range of Base station & 15 & $E_{f s}$ & $10 \mathrm{pJ} / \mathrm{bit} / \mathrm{m}^{2}$ \\
\hline 7 & $\mathrm{IE}_{\mathrm{sn}}=3$ joule & Initials Energy sensor nodes & 16 & Emp4 & $0.013 \mathrm{pJ} / \mathrm{bit} / \mathrm{m}^{4}$ \\
\hline 8 & $\mathrm{IE}_{\mathrm{pdp}}=5$ joule & Initials Energy PDP nodes & 16 & $E_{D A}($ Data Aggregation $)$ & $5 \mathrm{~nJ} / \mathrm{bit} /$ Signal \\
\hline 9 & $\mathrm{IE}_{\mathrm{tr} 1}=4$ joule & Initials Energy of Relay T1 & 17 & $E_{D C}($ Data Compression $)$ & $5 \mathrm{nj} / 10 \mu \mathrm{s} / 100 \mathrm{bit}$ \\
\hline
\end{tabular}

When a node receive this message it expends, $\boldsymbol{E}_{\boldsymbol{R} x}(\boldsymbol{l}) \boldsymbol{E n e r g y}$ and $\boldsymbol{E}_{\boldsymbol{R} x}(\boldsymbol{l})=\boldsymbol{l} * \boldsymbol{E}_{\text {lect }}$

\subsection{Result}

Table 5.a. List Radio Parameter and Values

Initially we took 55 Node, on $720 * 720$ square meter area, Applying SSPA (Scanning of Square Partion Algorithm) on the all senor node we have found the 8 node have required to replace by PDP After that again applying MADBS (Minimum Average Distance of Base Station for all PDP nodes) we found the location of Base Station is $(260,350)$. For this location the average distance from all PDP nodes is 191 meter which minimum. Now, applying SMTR2 algorithm we have found the required number Type 2 Relay Node is 10 and their Coordinates are shown in the following Screenshot and in table.
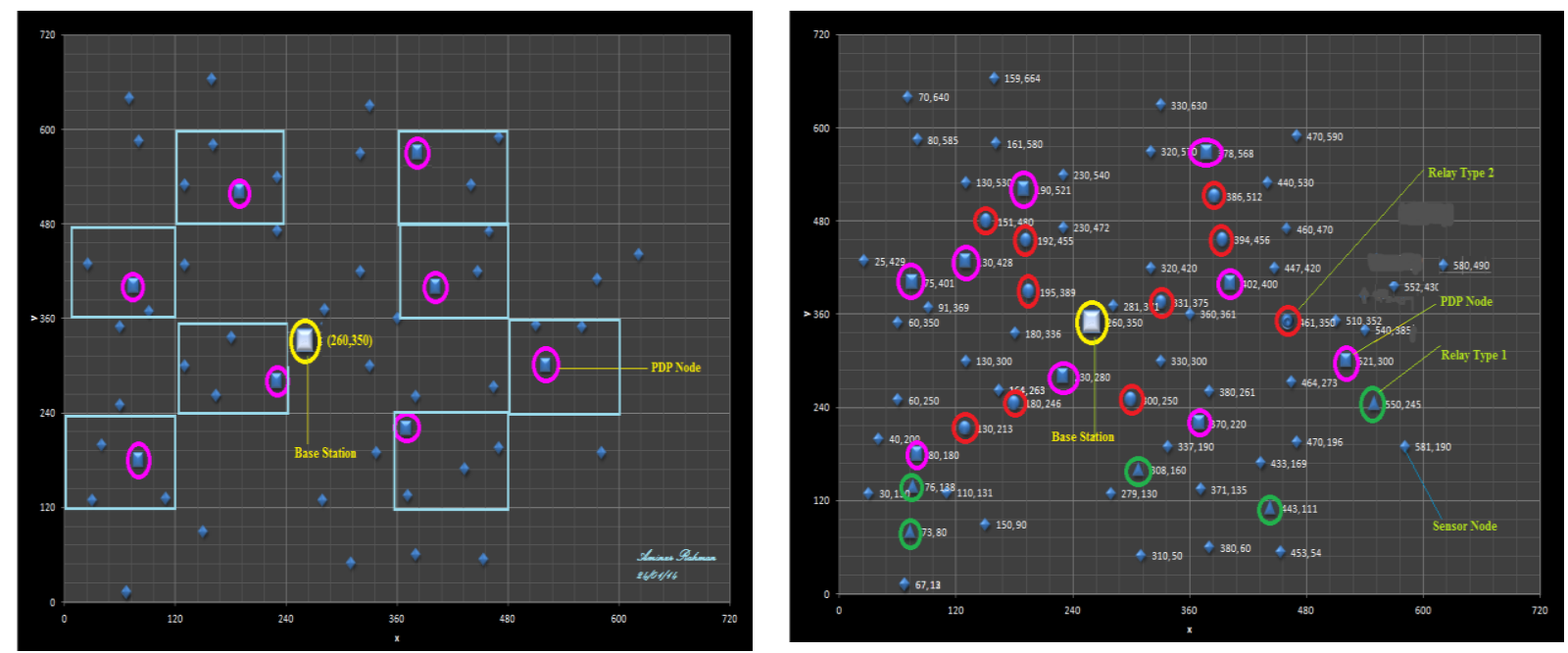

Fig 5.a \& 5.b.Showing selected PDP node, Optimal Location of Base Station, Type 1 and 2 Relay node 
Finally Applying SMTR1 algorithm we found that required number of Relay Node Type 1 is 5. So the total number of node is $(47+8+10+5)=70$ excluding base Station. Now applying Dynamic Technique (AEDC Protocol) on the above network we can get the dynamic architecture and behavior of the network, our proposed dynamic approach is AEDCP (Average Energy Dynamic Clustered Protocol). As we apply this protocol on the static network data Transmission will be start followed by Clustering phase and Data Transmission.

\subsection{1:Result of objective parameters}

\begin{tabular}{|l|l|l|l|l|l|}
\hline & $\begin{array}{l}\text { Total Energy Consumed } \\
\left.\text { (at 50 }^{\text {th }} \text { rounds }\right)\end{array}$ & $\begin{array}{l}\text { Live node } \\
\text { at 50 }^{\text {th }} \text { round) }\end{array}$ & Live nodes & degree(BEC) & Stability Period \\
\hline $\begin{array}{l}\text { Proposed } \\
\text { Approach }\end{array}$ & 230 joule & 10 & $\begin{array}{l}\text { Zero( after 64 } \\
\text { round })\end{array}$ & 36.17945007 & Up to 18 rounds \\
\hline BEEGP & 256 joule & 4 & $\begin{array}{l}\text { Zero( after 52 } \\
\text { rounds })\end{array}$ & 29.53337271 & Up to 12 rounds \\
\hline
\end{tabular}

Table 5.b. Showing the comparison of various objective parameters
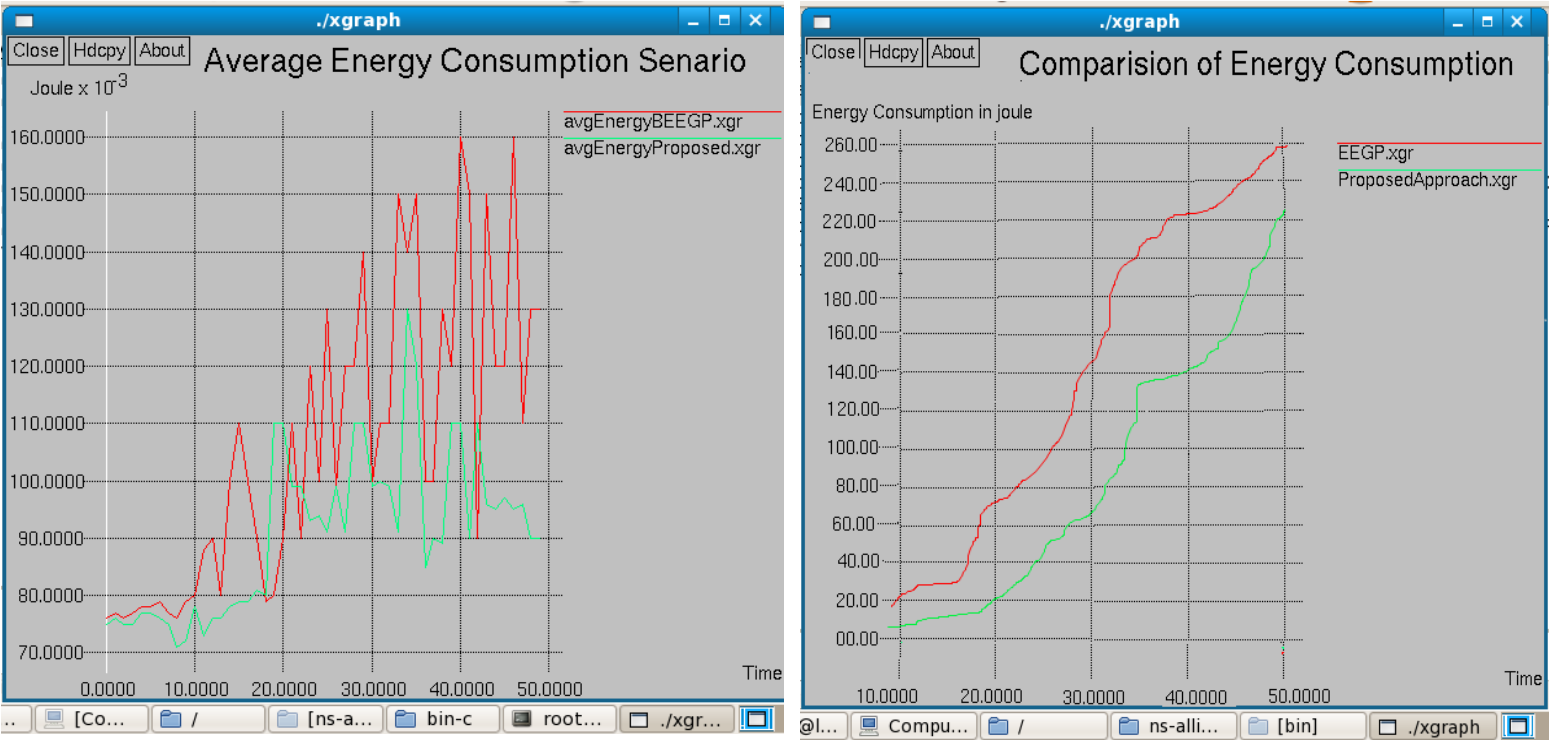

Fig 5.c \& 5.d. Showing the Average Energy Consumption and Total Energy Consumption Scenario
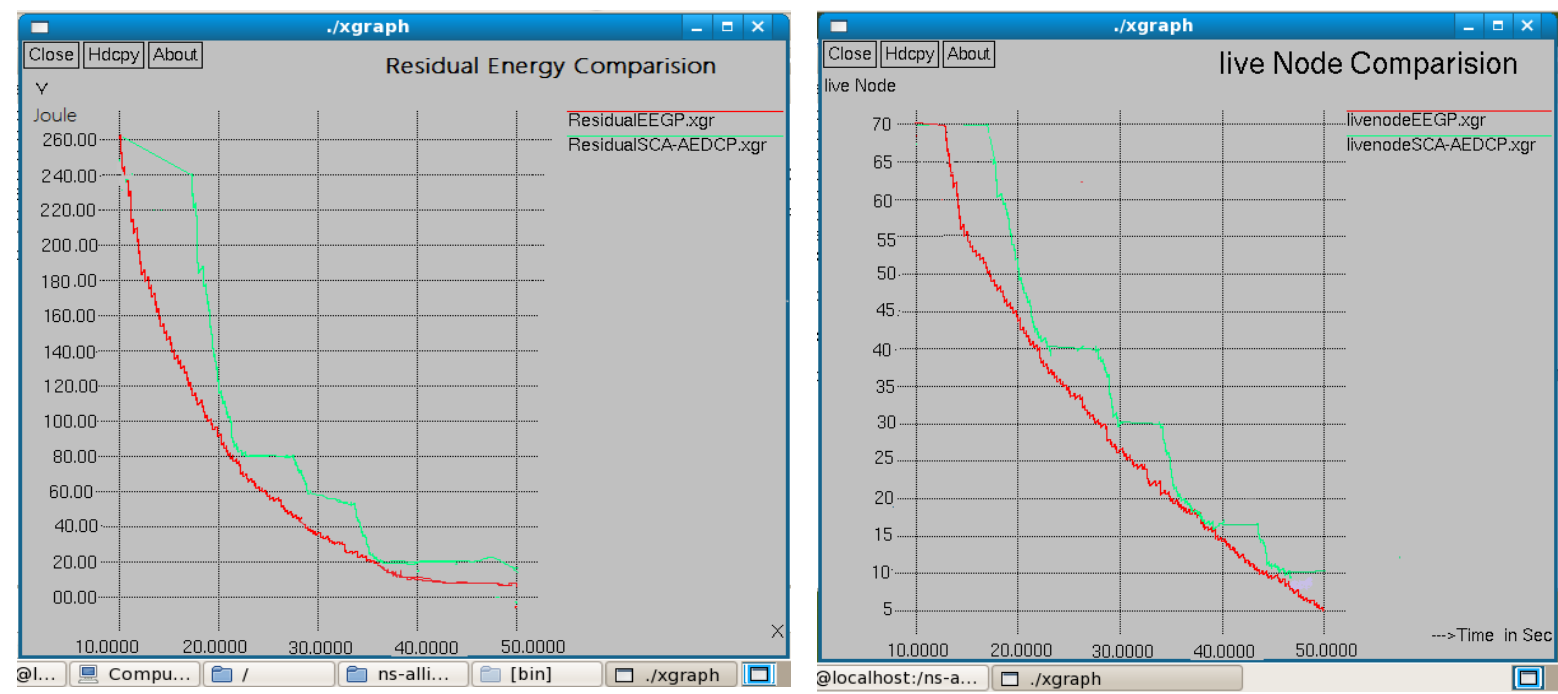

Fig 5.e \& 5.f.Showing Residual Energy Consumption scenario and Number of Live Nodes after every round. 


\subsubsection{Result of constrained parameters to compare the performance}

\begin{tabular}{|l|l|l|l|l|}
\hline & Average throughput[kbps] & Throughput[kbps] & Delay[ ms] & Pdf [receive/send ratio] \\
\hline Proposed Approach & 580.80 & Zero after 64 rounds & 11.781111 & 0.9182 \\
\hline BEEGP & 558.81 & Zero after 52 rounds & 15.435526 & 0.8451 \\
\hline
\end{tabular}

Table 5.c. Showing comparison of all constrained parameters

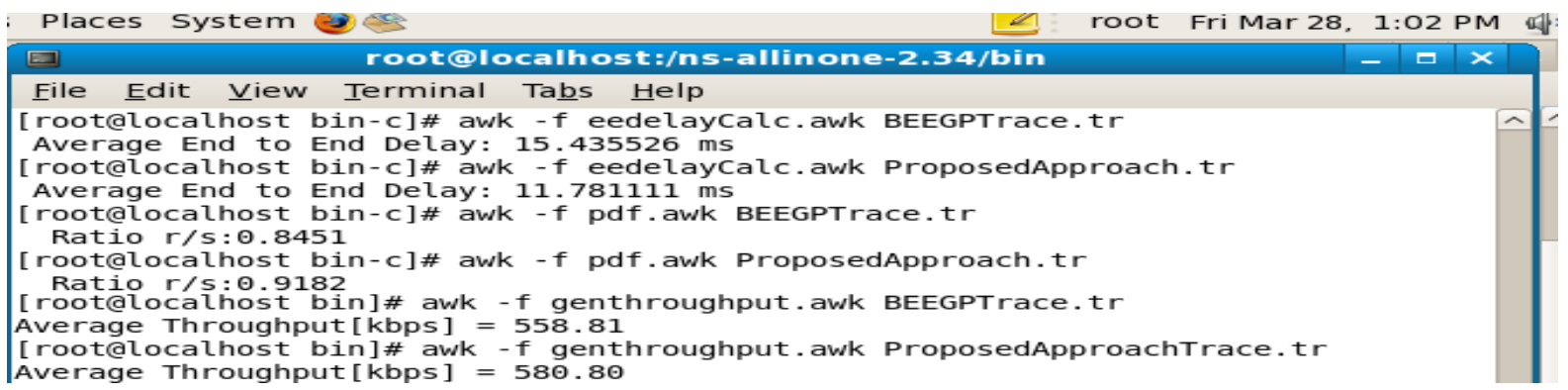

Fig 5.g: Showing simulated result of all constrained Parameters
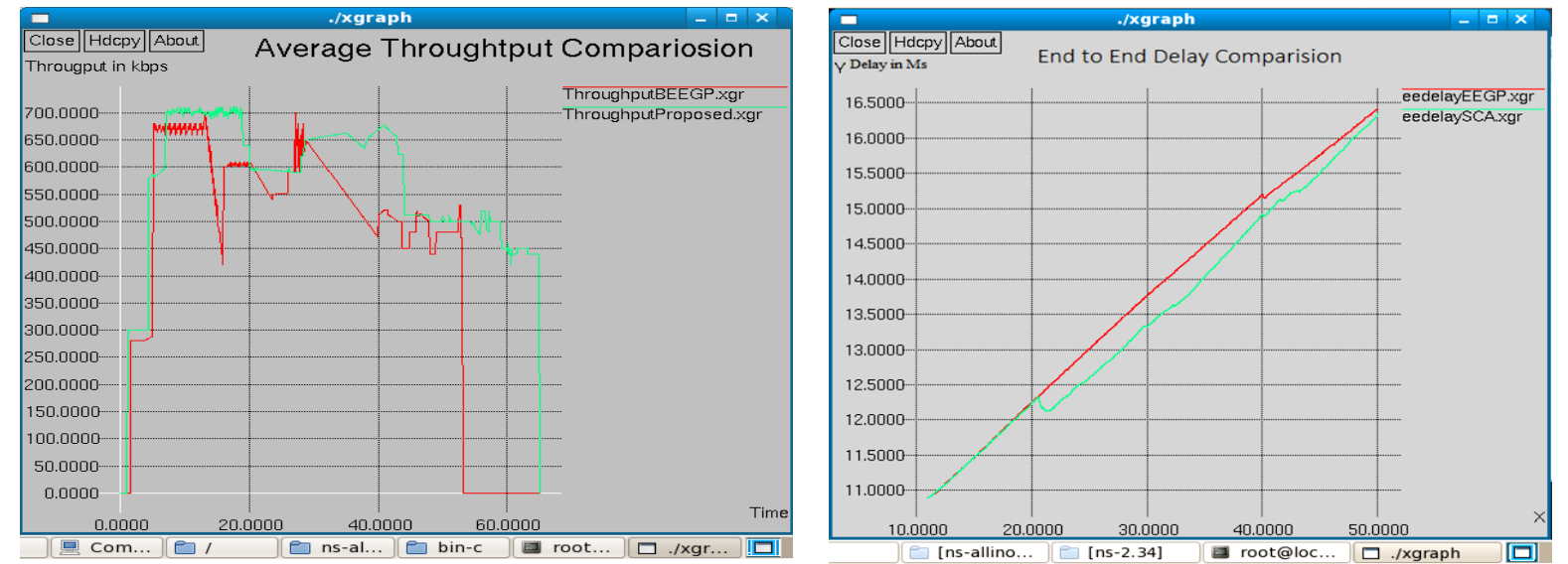

Fig 5.h \& 5.i.Showing the average throughput and average end to end delay scenario

\section{Discussions}

The Proposed Approach (SCA-AEDCP) is a Multi hop, Data Centric, Address Centric, and Conditional Multi Path Communication Protocol in Hierarchical Clustered Heterogeneous Wireless Sensor Network. We have compared this approach with similar protocol BEEGP (Balanced Energy Efficient Grouping Protocol). Although it has some similarities with the proposed approach we have also found some difference form theoretical studies. Besides of this, the simulation result has shown that overall performance of SCA-AEDCP approach is better than BEEGP for heterogeneous environments. The life time has increased by 12 rounds in Proposed Approach. And the values of the various constrained parameter have no needed to compromise. Average throughput increase by $21.99 \mathrm{kbps}$ and packet drop fraction is increase by 0.0731 . The stability period of proposed approach is 18 rounds; where in BEEGP is 12 rounds. The degree of Balance Consumption that 36.17 where this value in BEEGP is 29.15. The total energy consumption is reduced by 16 joule by up to 50 rounds in the proposed approach.

We can also highlight some theoretical comparison. First, this proposed approach takes advantages of Powerful Data Processing (PDP), As a result changing cluster head will not require very frequently. Secondly, it contains only one stage or two phases. That is Clustering Stage, which is again consisting of Cluster Setup phase and Data Transmission Phase, whereas BEEGP consists of Two Stage, Grouping Stage, and Data Transmission Stage. Further Data Transmission Stage consists of Cluster Setup phase. Third, First Cluster Setup phase will be completed on the basis average of total initial energy and later all the required cluster setup phase will be complete on the basis of average of total residual energy on that instant of time; whereas in BEEGP Grouping and Clustering is only on the basis of Average of total initial Energy. Fourth, Cluster member are not fixed for a particular cluster throughout the life time network, these are dynamic, and it changes the cluster 
during next cluster setup phase. where as in BEEGP Grouping stage and cluster setup phase occurred only once during the Life Time. So the clusters are Static and Cluster Members are fixed to a particular.

\section{Conclusion and Future Work}

Having seen the result of simulation of various parameters we can conclude that Systematic Combination of more than one technique can provides synergetic improvement to prolong life time sensor network. Since, increasing the life time of the network is based on lower energy consumption and higher degree of balanced consumption of energy. Lower energy consumption can be achieved by reducing the volume of data and the distance to be travel by data. So, it is somehow critical to achieve all the things by a single technique. So, again it is combination of arts and science to get better combination of many techniques. As, in our proposed approach, we have taken powerful data Processing node, Relay Type 1 and Type 2 Node to reduce the distance, powerful data node to reduce the volume of data and AEDCP for balance energy consumption. So, we have kept the name our approach as SCA-AEDCP (Systematic Combined Approach- Average Energy Dynamic Clustered Protocol). The advantages of the positive result of this work, is that it has open a new dawn towards many new research work in WSN to find which combination can give more better result. Since a single technique never can cover all issues, there is also a great importance to find out a best combination for different application. Although it has many advantages, but the limitation is, initials implementation has taken more computation time, and this is one drawback of such combined approach.

In future, we will implement this approach in a practical wireless sensor network tests bed to realize the practical applicability of its. We will also give a special importance to Important Junction Node to increase the life time it by increasing more the degree of balance consumption. Since it's a Combined Approach, it provides a new future scope to combine much technique to search for a better approach. SCA-AEDCP approach is basically engineered for those applications, which demand continuous data collection. In future we can adapt it for event detection by applying the concept active and sleep mode of the node for more energy saving.

\section{Acknowledgement}

This work has been supported by Department of Computer Engineering, Punjabi University Guru Kashi Campus, Punjab. For this, we are very thankful to Minakshi Bansal, Assistant Professor, for her valuable inspiration and suggestions.

\section{References}

[1]. S Taruna, Sakshi Shringi: A Cluster Based Routing Protocol for Prolonging Network Lifetime in Heterogeneous Wireless Sensor Networks, Published by International Journal of Advanced Research in Computer Science and Software Engineering, ISSN: 2277 128X, Volume 3, Issue 4, April,(2013).

[2]. Andreas Reinhardt, Daniel Burgstahler: Exploiting Platform Heterogeneity in Wireless Sensor Networks by Shifting Resource-Intensive Tasks to Dedicated Processing Nodes. In the Proceeding of $14^{\text {th }}$ IEEE International Symposium on a world of Wireless, Mobile and Multimedia Networks (Wow Mom), IEEE Press, June, (2013).

[3]. Adamu Murtala Zungeru et al. "Energy Efficiency Performance Improvements for Ant-Based Routing Algorithm in Wireless Sensor Networks" Hindawi Publishing Corporation Journal of Sensors, (2013).

[4]. Jiau-Jian-Liaw, Lin-Haung Chang, Hung Chi Chu; "Improving Life Time in Heterogeneous Wireless Sensor Network with energy Efficient Grouping Protocol", Publish at International journal of Innovative Computing, Information and Control, Volume 8, number 9,(2012).

[5]. Fabio Engel et al., "Relay Selection Methods for Maximizing the Lifetime of Wireless Sensor Networks", presented on IEEE Wireless Communication and Networking Conference (WCNC), (2013).

[6]. Mark Yarvis, Nandakishore Kushalnagar, "Exploiting Heterogeneity in Sensor Networks". Intel Research and Development, Portland State University 2111 N.E. 25th Ave., Hillsboro, (2011).

[7]. Mohammadreza Eslaminejad, Shukor Abd Razak; "Fundamental Lifetime Mechanisms in Routing Protocols for Wireless Sensor Networks: a Survey and Open Issues, published on Sensors, "www.mdpi.com", (2012).

[8]. Mei Wu: Energy Efficient Routing Protocol in Heterogeneous Wireless Sensor Network, A PhD thesis directed by DR. MARTIN COLLIER at Dublin City University, (2012).

[9]. Jonathan Gana Kolo et al.; An Adaptive Lossless Data Compression Scheme for Wireless Sensor Networks, Hindawi Publishing Corporation Journal of Sensors, Article ID 539638, 20 pages ,(2012).

[10]. V. God bole, FCA - An Approach On LEACH Protocol Of Wireless Sensor Networks Using Fuzzy Logic, International Journal of Computer Communications and Networks (IJCCN), 2(3), pp. 1-13, (2012)

[11]. Jing-hui Zhong and Jun Zhang: Ant Colony Optimization Algorithm for lifetime Maximization in Wireless Sensor Network with Mobile Sink. Philadelphia, Pennsylvania, USA. July 7-11, (2012).

[12]. Pagun Park "Modelling, Analysis, and Design of Wireless Sensor Network protocol" Doctoral thesis KTH School of Electrical Engineering Automatic Control Lab SE-100 44. Stockholm Sweden TRITA-EE 2011:001 ISSN 1653-5146, (2009).

[13]. Ewa Niewiadomska et.al. "Comparative Study of Wireless Sensor Networks Energy-Efficient Topologies and Power Save Protocols", published on journals of telecommunication and information technology, (2009).

[14]. Shi, Y. and Hou, Y. T: Optimal base station placement in wireless sensor networks, Published by ACM Transaction on Sensor Network. 5, 4 Article 32. November, (2009).

[15]. Ying Zhu, Qi Han, “The More Relay Nodes, the More Energy Efficient?” published in the proceeding of IEEE CSE'09, 12th IEEE International Conference on Computational Science and Engineering, Vancouver, BC, Canada, August 29-31, (2009).

[16]. Gao, D.; Liang, L.; Gong, Y.; Zhang; “ Micro Sensor Multi-Path Routing Protocol in Wireless Sensor Networks”, In Proceedings of the International Symposium on Multimedia Technology, (CNMT '09), Wuhan, Hubei, China, 18-20 January, (2009). 
[17]. Hong, L.; Yang, J; “An Energy-Balance Multipath Routing based on Rumor Routing for Wireless Sensor Networks”. In Proceedings of the Fifth International Conference on Natural Computation, (ICNC '09), Tianjin, China, 14-16 August, (2009).

[18]. Wang, Z.; Bulut, E.; Szymanski, B.K. "Energy Efficient Collision Aware Multipath Routing for Wireless Sensor Networks". In Proceedings of the IEEE International Conference on Communications (ICC’09), Dresden, Germany, 14-18 June, (2009).

[19]. Okdem, S.; Karaboga, D. "Routing in wireless sensor networks using an ant colony optimization (ACO) router chip", Sensors, (2009)

[20]. Eghbali et al., "Energy Efficient Load-Balanced Multi-Sink Routing Protocol for Wireless Sensor Networks", In Proceedings of the 10th International Conference on Telecommunications (Con TEL '09), Zagreb, Croatia, 8-10 June, (2009).

[21]. Ranganathan Vidhyapriya1, Ponnusamy Vanathi; “Energy Efficient Data Compression in Wireless Sensor Networks", the International Arab Journal of Information Technology, Vol. 6, No. 3, July, (2009)

[22]. G. Xing, R. Tan, B. Liu, J. Wang, X. Jia, and C.-W. Yi, "Data fusion improves the coverage of wireless sensor networks," in the Proceedings of the 15th Annual International Conference on Mobile Computing and Networking (ACM Mobile Com 2009), pp. 157168, Beijing, China, 20-25 September (2009).

[23]. Saad, E.M. et al: "Energy-aware gathering strategy for wireless sensor networks". Int. J. Distributed. Sensor. Network, (2009).

[24]. Issariyakul .Terawatt, Husain. Ekram, "Introduction to Network Simulator 2", published on Springer Science+ Business Media, LLC, 233 Spring Street, New York, NY10013, USA, (2009)

[25]. Ghasem Aghaei et al, "Ant Colony-Based Many-to-One Sensory Data Routing in Wireless Sensor Networks". In Proceedings of the International Conference on Computer Systems and Applications (AICCSA '08), Doha, Qatar, 31 March-4 April (2008).

[26]. Soo Kim, Jeong-Gil KO, Jongwon Yoon and Heejo Lee, "Multiple-Objective Metric for Placing Multiple Base Stations in Wireless Sensor Networks" (C) IEEE, (2007).

[27]. Rajagopalan, Ramesh and Varshney, Pramod K., "Data aggregation techniques in sensor networks: A survey" Electrical Engineering and Computer Science, (2006).

[28]. Chen G., Guo T., Wang W., Zhao T. (2006), “Ant-Based Routing Protocol in Wireless Sensor Networks"1-4244-0429-0-/06,IEEE, (2006).

[29]. Y. Thomas Hou, et.al, "On Energy Provisioning and Relay Node Placement for Wireless Sensor Network"; IEEE Transaction on Wireless Communication, VOl 4, No. 5, September, (2005).

[30]. V. Mhatre and C. Rosenberg, "Homogeneous vs. Heterogeneous Clustered Sensor Networks: A Comparative Study", 2004 IEEE International Conference on Communications (ICC 2004), Paris France, June, (2004).

[31]. Gabber E., Smith M. A. "Trail Blazer: A Routing Algorithm Inspired By Ants", IEEE International Conference on Network Protocols (ICNP'04), (2004).

[32]. De, S.; Qiao, C.; Wu, H, "Meshed multipath routing with selective forwarding: an efficient strategy in wireless sensor networks". Computer. Network. 43, 481-497; (2003).

\section{Authors' Biographies:}

Aminur Rahman received B.Tech degree from Punjab Technical University; Jalandhar, India in 2010 and the

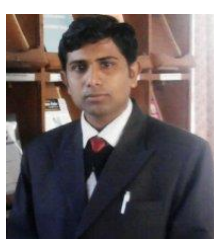
M.Tech degree from Punjabi University Guru Kashi Campus, Punjab, India. He started working as a lecturer at the MIMIT (Est. by Govt. of Punjab), Malout and later he also worked at JSS Institute of Engineering \& Technology, Patiala, Punjab. He is currently working an Assistant Professor in the Department of Computer Science \& Engineering at Gulzar Institute of Engineering \& Technology, Punjab, India. His interest research areas include Wireless Sensor Network, Biologically inspired Algorithm, and Database Management. He has published two research papers on international journal and one on national journal.

Rajbhupinder Kaur received her M.Tech Degree from Punjabi University; Patiala, India in 2010 and B.Tech

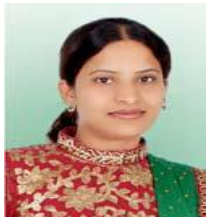
degree from Punjab Technical University, Jalandhar in 2006. She started working as a lecturer at Lala Lajpat Rai Institute of Engineering\& Technology, Moga, and Punjab. Currently she is working as an Assistant Professor in the Department of Computer Engineering at Punjabi university Guru Kashi Campus, Talwandi Sabo, Punjab. Her research interests are in the fields of Mobile Ad-Hoc Network, Network Security, Nanotechnology, wireless sensor networks. She has published many national \& international papers.

Sanjib Das is working as a Technology Analyst at Infosys Technology, Pune, India. He received his B.Tech and

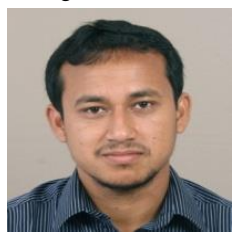
M.Tech degree in computer science from Punjab Technical University and IIT, Guwahati respectively. He has qualified GATE examination several times. He has a passion for network security and worked on the field for last two years. During his M.Tech, he proposed an Intrusion Detection system for DNS Cache Poisoning Attack. Apart from network security, he works on application software and web development. He has published many research paper on international and national journal. 